\title{
A New Index for Detecting Frequency of Unknown Underwater Weak Signals with Genetic Algorithm
}

\author{
Weixiang Yu, Xiukui Li \\ School of Information and Communication Engineering \\ Dalian University of Technology, China
}

\begin{abstract}
In this paper, a new index is proposed for detecting the frequency of unknown underwater signals based on the stochastic resonance theory. When the received weak signal is input into the stochastic resonance system, first, by frequency analysis, the frequency with the highest amplitude $A_{m}$ of the output signal spectrum is considered as the pre-detection frequency. Then a cosine signal with the pre-detection frequency and unit amplitude is constructed. Define the pre-signal-to-noiseratio as the logarithm of the squared amplitude $A_{m}$ over the mean of signal amplitudes in all other frequencies. The new index is defined as the product of the pre-signal-to-noise-ratio and the correlation coefficient between the received unknown signal and the constructed cosine signal. The new index is featured by taking into account the signal characteristics in both time and frequency domain, and it will yield better signal frequency detection performance. In addition, to improve the time efficiency of the frequency detection, a method to bound the searching range, keyed to the genetic algorithm, of the stochastic resonance system parameters is proposed. The method can be used to detect the frequency of both single frequency and frequency-hopping unknown signals. With the designed new index and system parameter bounding method, the simulations and experiments for the weak underwater unknown signals are conducted. Compared to the piecewise mean value index and weighted power spectral kurtosis index, the new index yields a higher detection probability at varied input signal-to-noise ratios and signal frequencies. With bounding system parameter searching ranges, the time efficiency is improved. The main purpose of this paper is to detect the frequency of unknown underwater weak signals by stochastic resonance system with genetic algorithm. The main contributions are summarized as follows. First, the detection probability of weak signals is improved by stochastic resonance system with the proposed signal detection index than some other indexes. Second, to improve the time efficiency of the signal frequency detection, a method to bound the searching range of system parameters is proposed.
\end{abstract}

Keywords-Stochastic resonance; underwater weak signal detection; genetic algorithm; frequency detection; frequency-hopping signal; index

\section{INTRODUCTION}

The detection and identification of underwater unknown targets are of great significance for the coastal defense development. However, because of natural and human activities, the underwater environment is very complex, such as wind and waves on the sea surface, marine biological activities, ocean currents on the seabed and the movement of hulls. In addition, some underwater targets can change their frequencies and other information to hide themselves. These factors make the detection and identification of underwater targets more challenging. Therefore, the efficient detection of underwater targets in such a complex environment is very important for both scientific research and engineering practice. The methods of traditional weak signal detection usually use finite impulse response (FIR) and infinite impulse response (IIR) [1], [2] filters to filter out the background noise mixed with the signal. Although these methods have some effects on filtering the out-of-band noise of signal, they will fail when the noise is distributed in the signal band. Stochastic resonance (SR) [3] theory is a weak signal detection method with high efficiency, which is different from traditional signal detection methods. With the SR system, the weak signal can be enhanced, and the system output signal-to-noise ratio $\left(S N R_{o}\right)$ will be maximized by utilizing the background noise. The SR system is mainly composed of weak signal, background noise, and nonlinear system [4]. SR theory was first proposed by R.Benzi et al. in 1981 [5], which explained a phenomenon that the glacial and warm climates occurred periodically in ancient climate. Then SR theory has been further developed. Nowadays, it has been applied extensively to many subjects such as meteorology [6], hydroacoustics [7], biomedicine [8] and mechanical mechanics [9], [10].

In recent years, researchers have paid more attention to the field of weak signal detection based on SR theory. Wang et al. The author in [11] extend the bistable SR system to the tri-stable system by adjusting the three potential heights of SR system potential function. They use the differential evolutionary particle swarm algorithm to search the optimal values of system parameters, which can effectively improve the system $S N R_{O}$ by simulations and experiments. Zheng et al. The author in [12] propose a fractional-order stochastic resonance (FOSR) multi-parameter optimization algorithm based on genetic algorithm (GA), which is beneficial to the application and popularization of FOSR in weak signal detection. H. T. Reda et al. The author in [13] discuss the application of SR in spectrum sensing and propose a firefly-inspired algorithm to optimize the SR and noise parameters of the dynamic system to improve signal detection. Guo et al. [14], [15] detect the multi-frequency weak signal by the cascading and paralleling of SR system. Based on the adiabatic approximation theory [16], SR theory is applicable to the signal detection of low frequency $(\ll 1 \mathrm{~Hz})$. However, most of the signal frequencies in practical applications are not low. Leng et al. The authors in [17], [18] propose to transform high frequency to low frequency signals by using scale transformation and secondary sampling theory such that the high frequency weak signals can be detected based on SR theory. Ji et al. [19] realize the detection of hydroacoustic high frequency chirp signals by SR system, and verify the correctness and feasibility of this method by simulations and experiments. The performance of SR system is directly related to the detection probability 
of weak signal. It is crucial to design a index with high adaptability for weak signal detection, which can keep the resonance response to follow the unknown signal features. The $S N R_{O}$ can be increased by SR system, hence $S N R_{o}$ and signalto-noise ratio gain $\left(S N R_{g}\right)$ [20], [21] are the most common and intuitive indexes for measuring the performance of the adaptive SR system. However, to calculate $S N R_{o}$ and $S N R_{g}$, some prior information should be available, such as the frequency of weak signal. Normally, these prior information cannot be obtained in practical applications. It is indispensable to define a new index for the detection of completely unknown signals. Therefore, many new indexes have been proposed, such as weighted kurtosis index [22], time domain correlated kurtosis index [23], correlation coefficient index [24], weighted power spectrum kurtosis (WPSK) index [25], entropy kurtosis variation product index [26] and piecewise mean value index (PMV) [27]. Although these indexes are feasible in some conditions, their adaptability is constrained. For example, for some single indexes, the time and frequency domain characteristics of the signal cannot be taken together to detect the signal. For some multiple indexes, they are sensitive to signal frequency and pulse signal. When the input signal frequency is high or the input signal contains a large amount of pulse signals, with these indexes the weak signal detection probability is low. In this paper, a new index is proposed to improve the weak signal detection probability by SR system.

The contributions of this paper are as follows. First, a new index is designed for weak signal detection and GA is used as the optimization method to search the optimal values of system parameters. The signal detection probability with the new index is proved to be higher than that by SR systems with PMV and WPSK indexes through simulations and experiments. The designed new index takes into account both the time and frequency domain characteristics of the signal, and it is insensitive to the change of signal frequency. Second, to decrease the frequency detection time for both single frequency and frequency-hopping signals, a method to bound the searching ranges of system parameters is proposed. However, there are some limitations in the existing work. The experimental site is located at the seaside of Xing-hai Park in Dalian City in this paper, which is different from the deep sea. In addition, the simulation is carried out in Gaussian noise, which is different from the actual noise type.

The remaining parts of this paper are organized as follows. The SR theory and the definition of a new index are introduced in Section II. For single frequency and frequency-hopping signals, the method to bound the searching ranges of system parameters $a$ and $b$ is presented in Section III. The simulations and experiments are analyzed in Section IV and V. The results and discussion are introduced in Section VI. The future plans and improvements are introduced in Section VII. Finally, this paper is concluded in Section VIII.

\section{NeW IndeX Definition AND Design of SR System}

\section{A. Stochastic Resonance Theory}

The bistable SR system can be given by nonlinear Langevin equation [28],

$$
\frac{d x}{d t}=-V^{\prime}(x)+s(t)+n(t)
$$

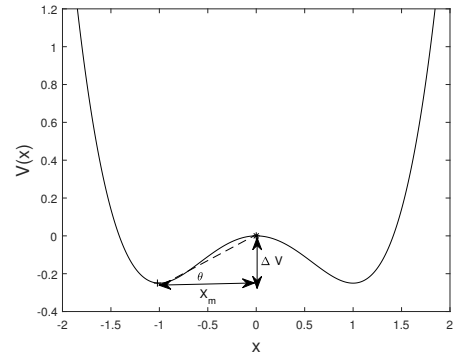

Fig. 1. The Potential Function Curve of $V(x)$, when $a=b=1 . \Delta V$ is the Potential Height, $x_{m}$ is the Half Width of Potential well, $\tan \theta=\Delta V / x_{m}$.

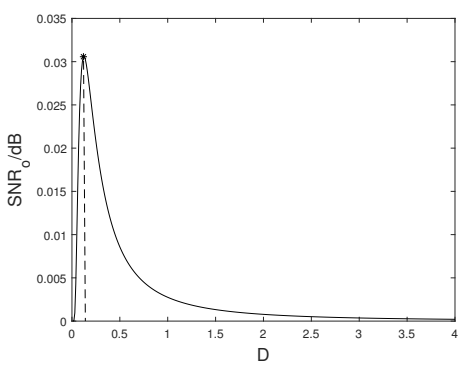

Fig. 2. The Curve of $S N R_{O}$ with Noise Intensity $D$, when $A=0.1, a=b=$ 1 .

where $V(x)=-\frac{a}{2} x^{2}+\frac{b}{4} x^{4}$ is the potential function and its curve is shown in Fig. 1. $x$ is the SR system output signal. System parameters $a$ and $b$ are real numbers greater than zero. $s(t)=A \cos \left(2 \pi f_{0} t\right)$ is the weak periodic signal to be detected, $A$ is the signal amplitude and $f_{0}$ is the frequency. $n(t)$ is the noise, and $n(t)=\sqrt{2 D} \varepsilon(t) . D$ is the noise intensity and $\varepsilon(t)$ represents the Gaussian white noise with zero mean and variance one.

The SR system is in a stable state and the particle is in one of the two potential wells when there is no external signal input to the system. When an appropriate noise is input to the system, the particle will obtain energy from the noise and then skip the potential barrier to complete the transition between the two potential wells. Because the voltage difference between the two bistable potential wells is much larger than the original input signal amplitude, the $S N R_{O}$ of SR system is greatly improved in this situation. The $S N R_{O}$ of SR system is given by

$$
S N R_{o} \approx \sqrt{2} \Delta V\left(\frac{A}{D}\right)^{2} e^{-\frac{\Delta V}{D}}
$$

where $A$ is the amplitude of system input signal $s(t), \Delta V$ is the potential height of the potential function. The $S N R_{O}$ with respect to the noise intensity $D$ is shown in Fig. 2.

It is inefficient and lacks of adaptivity to put the nonlinear system in a resonant state by adjusting noise intensity [29]. The potential height $\Delta V$ determined by the system parameters $a$ and $b$ is crucial for the particle to complete the periodic transition between the two potential wells. Parameters $a$ and $b$ can be searched by some optimization methods. In this paper, we choose GA as the optimization method to search 
the optimal values of system parameters dynamically [30].

To detect the signal frequency by SR system, it is required that the input signal be of low frequency. A signal with high frequency can be down-converted into a low frequency signal by re-sampling the original signal with a ratio $R$. Then the low frequency $f_{0}^{\prime}$ of weak signal can be detected by SR system and finally we can restore the actual signal frequency by $f_{0}=$ $f_{0}^{\prime} \times R$.

\section{B. Definition of the New Index}

For the frequency detection of unknown signals, various indexes have been proposed such as PMV and WPSK indexes. However, the signal frequency detection probability with these indexes are low. Herein, we propose a new index for the signal frequency detection through a SR system. Denote the received unknown weak signal by $r(t)$ and $r(k)$ is its sampled one signal. The new index is designed as follows.

I). The output signal $x(k)$ of SR system can be calculated by the fourth-order Longe-Kutta algorithm. The Fourier transform of $x(k)$ is denoted by $H(f)$. The frequency with the maximum amplitude of $H(f)$ is denoted by $f_{m}$.

II). Construct a new cosine signal $s^{\prime}(t)=\cos \left(2 \pi f_{m} t\right)$ with frequency $f_{m}$ and initial phase zero. The absolute value of the correlation coefficient between $r(t)$ and $s^{\prime}(t)$ is written as $C=\left|R\left(r(k), s^{\prime}(k)\right)\right|$, where $r(k)$ and $s^{\prime}(k)$ are the sampling signals of $r(t)$ and $s^{\prime}(t)$, respectively.

III). The pre-signal-to-noise ratio of the output signal $x(k)$ is defined as $P S N R=10 \times \log _{10} \frac{H^{2}\left(f_{m}\right)}{\sum_{f \neq f_{m}} H(f) / N}$, where $H\left(f_{m}\right)$ is the peak amplitude of $H(f)$ and $N$ is the length of output signal $x(k)$.

IV). The new index, called CSNR, is defined as the product of $C$ and $P S N R$, and the value of CSNR can be calculated by $\gamma_{c s n r}=C \times P S N R$, which will be used as the fitness function of GA for searching the optimal values of system parameters $a$ and $b$.

\section{Process Detecting the Frequency of Weak Signals with the New Index}

The sampled signal $r(k)$ of received unknown weak signal $r(t)$ is input to the optimal SR system and its spectrum $H(f)$ is obtained. The frequency with the maximum spectrum amplitude is considered as the frequency of $r(t)$. The frequency detecting process of the optimal SR system for detecting the frequency of weak signals is shown in Fig. 3.

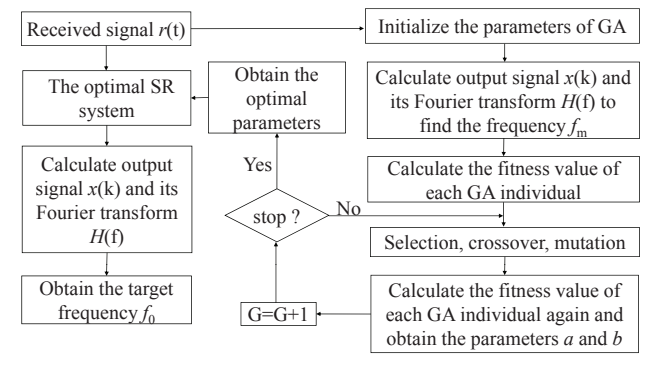

Fig. 3. The Frequency Detecting Process of SR System.
When the frequency with the maximum spectrum amplitude is not the target signal frequency $f_{0}, s^{\prime}(t)$ and $r(t)$ will not be highly correlated, and the value of $C$ will be smaller. Hence, $C$ can be used as a single time domain index. In addition, when the value of $P S N R$ is larger, the peak amplitude of output signal spectrum is larger than other amplitudes obviously, which can reduce the influence of other random frequencies on the frequency with the maximum spectrum amplitude. Hence, the value of $P S N R$ can be used as a single frequency domain index. To jointly consider both the time and frequency domain characteristics of the output signal, the product of the two single indexes can be a new index, which will yield better detection performance. When $n(t)$ is zero mean Gaussian noise, the correlation coefficient between $r(t)$ and $s^{\prime}(t)$ is

$$
C=\left|R\left(r(k), s^{\prime}(k)\right)\right|=\frac{\left|\operatorname{Cov}\left(r(k), s^{\prime}(k)\right)\right|}{\sqrt{\operatorname{Var}(r(k)) \times \operatorname{Var}\left(s^{\prime}(k)\right)}}
$$

The denominator of (3) is a constant and its numerator is

$$
\begin{aligned}
c & =\left|\sum_{k=1}^{N} s(k) \times s^{\prime}(k)+\sum_{k=1}^{N} n(k) \times s^{\prime}(k)\right| \\
& =\left\{\begin{array}{l}
c_{1}, f_{m}=f_{0} \\
c_{2}, f_{m} \neq f_{0}
\end{array}\right.
\end{aligned}
$$

Generally, $c_{1} \geq c_{2}$, where $c$ is the numerator of (3), $s(k)$, $s^{\prime}(k)$ and $n(k)$ are the sampled signal of $s(t), s^{\prime}(t)$ and $n(t)$, respectively. $N$ is the number of signal $s(k)$. When $f_{m}=f_{0}$, $c=c_{1}$, otherwise, $c=c_{2}$.

\section{A Method to Bound the Searching Ranges of SYSTEM PARAMETERS $a$ AND $b$}

\section{A. The Searching Range of System Parameter $b$ for Single Frequency Signal Detection}

The intensity of noise added to the nonlinear system will affect SR system operation. If the noise intensity is too low, the particle cannot obtain enough energy from the noise to skip the potential barrier. If the noise intensity is too high, although the particle can obtain enough energy from the noise to skip the potential barrier and resonance will occur, there will be a large amount of random noise mixed with the output signal. In this situation, the $S N R_{O}$ of SR system is still very low and the signal frequency cannot be detected from the output signal with the strong background noise. Different potential heights $\Delta V$ determined by system parameters $a$ and $b$ have different fitness values of GA. The optimal values of system parameters $a$ and $b$ can be obtained by GA with the CSNR index. To reduce the searching time of system parameters $a$ and $b$, we propose a method to limit the searching range of parameter $b$ for GA.

From Fig. 1, the half width of potential well $x_{m}=\sqrt{\frac{a}{b}}$ and the potential height $\Delta V=\frac{a^{2}}{4 b}$ [31]. The critical conditions for the particle to skip the potential barrier are given by

$$
\left\{\begin{array}{c}
\frac{\partial V(x, t)}{\partial x}=-a x+b x^{3}=0 \\
\frac{\partial^{2} V(x, t)}{\partial x^{2}}=-a+3 b x^{2}=0
\end{array}\right.
$$


Where $x$ is the system output signal. Hence, the critical amplitude of particle is $A_{c}=\sqrt{\frac{4 a^{3}}{27 b}}$, which is considered as the threshold for the particle to skip the potential barrier. The amplitude of signal and noise intensity need to satisfy

$$
\left\{\begin{array}{l}
A \leq A_{c} \\
A_{c} \leq A+D
\end{array}\right.
$$

When $A>D$, the input signal-to-noise ratio $\left(S N R_{i}\right)$ of system is high, and we can obtain the signal frequency from the received signal spectrum easily. Hence, assume $A \leq D$, from (6), we can obtain $b \geq \frac{4 a^{3}}{27(A+D)^{2}} \geq \frac{4 a^{3}}{27(2 D)^{2}}$, where $A$ is the amplitude of input signal $s(t)$, and $D$ is the noise intensity. When the standard deviation of noise $n(t)$ is $\sqrt{2 D}$, hence, we can obtain the received signal power $P \approx 2 D$. Therefore, $b \geq \frac{4 a^{3}}{27 P^{2}}$ at low $S N R_{i}$, which indicates the potential height $\Delta V$ determined by the system parameters $a$ and $b$ would not be large. In addition, $\Delta V$ would not be small, otherwise, there will be a large amount of random noise mixed with the output signal. In this situation, the frequency $f_{0}$ of the target signal cannot be distinguished from other random frequencies. Hence, we can set a searching range for the parameter $b$. Define $\tan \theta=\frac{\Delta V}{x_{m}}=\frac{a \sqrt{a}}{4 \sqrt{b}}$ (see Fig. 1 for $\theta$ ). Therefore, we can obtain the searching range of parameter $b$, where $P$ is the power of the received signal

$$
\frac{4 a^{3}}{27 P^{2}} \leq b \leq \frac{a^{3}}{16(\tan \theta)^{2}}
$$

\section{B. The Searching Ranges of System Parameters $a$ and $b$ for} Frequency-Hopping Signal Detection

To detect the frequency of frequency-hopping signal by the proposed SR system and reduce the searching time of parameters $a$ and $b$, we will constrain the searching ranges of $a$ and $b$. The Kramers rate $r_{k}$ is the twice of signal frequency $f_{0}$ when resonance occurs [32].

$$
r_{k}=\frac{a}{\sqrt{2} \pi r} e^{-\frac{a^{2}}{4 b D}}=\frac{a}{\sqrt{2} \pi r} e^{-\frac{\Delta V}{D}}=2 f_{0}
$$

where $r$ is the damping factor of the second order duffing equation in (9) and $r_{k}$ is the Kramers rate.

$$
\frac{d^{2} x}{d t^{2}}-r \frac{d x}{d t}=-V^{\prime}(x)+s(t)+n(t)
$$

where $x$ is the system output signal, $s(t)$ is the input signal, and $n(t)$ is the background noise.

The potential height $\Delta V$ affects the transition of the particle between the two potential wells and then the accuracy of signal frequency detection. Derived from (8), the relationship between the frequency $f_{0}$ and potential height $\Delta V$ is (10). Hence, $\Delta V$ needs to change while the input signal frequency changes. $\Delta V$ should decrease to help the particle to complete the transition between the two potential wells when the signal frequency $f_{0}$ increases. When the signal frequency $f_{0}$ decreases, $\Delta V$ should increase to reduce the speed of particle transition between the two potential wells, which can make the output signal frequency decrease and let it equal to the weak input signal frequency.

$$
f_{0}=\frac{a}{2 \sqrt{2} \pi r} e^{-\frac{\Delta V}{D}}
$$

The relationship between the values of two adjacent signal frequencies is $\frac{f_{b}}{m}<f_{c}<m \times f_{b}$, where $m$ is a real number greater than zero. $f_{b}$ is the signal frequency before the frequency changes, and the current frequency is $f_{c}$. Hence,

$$
\frac{f_{b}}{f_{c}} \in\left(\frac{1}{m}, m\right)
$$

Set $\Delta V_{n}=\frac{\Delta V_{c}-\Delta V_{b}}{D}$, where $\Delta V_{b}$ is the optimal potential height before the frequency changes and $\Delta V_{c}$ is the optimal potential height for the current frequency. Hence,

$$
\Delta V_{n} \in\left(\ln \left(\frac{a_{c}}{m \times a_{b}}\right), \ln \left(\frac{m \times a_{c}}{a_{b}}\right)\right)
$$

where $a_{b}$ is the optimal value of parameter $a$ before the frequency changes and $a_{c}$ is the optimal $a$ for the current frequency. When $f_{0}$ increases,

$$
\left\{\begin{array}{l}
\Delta V_{n} \in\left(\ln \left(\frac{a_{c}}{m \times a_{b}}\right), 0\right), \frac{a_{b}}{m} \leq a_{c}<m \times a_{b} \\
\Delta V_{n} \in\left(\ln \left(\frac{a_{c}}{m \times a_{b}}\right), \ln \left(\frac{m \times a_{c}}{a_{b}}\right)\right), a_{c}<\frac{a_{b}}{m} \\
0, a_{c} \geq m \times a_{b}
\end{array}\right.
$$

When $f_{0}$ decreases,

$$
\left\{\begin{array}{l}
\Delta V_{n} \in\left(0, \ln \left(\frac{a_{c}}{m \times a_{b}}\right)\right), \frac{a_{b}}{m} \leq a_{c}<m \times a_{b} \\
\Delta V_{n} \in\left(\ln \left(\frac{a_{c}}{m \times a_{b}}\right), \ln \left(\frac{m \times a_{c}}{a_{b}}\right)\right), a_{c} \geq m \times a_{b} \\
0, a_{c}<\frac{a_{b}}{m}
\end{array}\right.
$$

Generally, when $\frac{a_{b}}{m} \leq a_{c}<m \times a_{b}$

$$
\left\{\begin{array}{l}
\Delta V_{n} \in\left(\ln \left(\frac{a_{c}}{m \times a_{b}}\right), 0\right), \text { if } f_{0} \text { increases } \\
\Delta V_{n} \in\left(0, \ln \left(\frac{a_{c}}{m \times a_{b}}\right)\right), \text { if } f_{0} \text { decreases }
\end{array}\right.
$$

Hence, when $f_{0}$ increases,

$$
\Delta V_{c} \in\left(\Delta V_{b}+D \times \ln \left(\frac{a_{c}}{m \times a_{b}}\right), \Delta V_{b}\right)
$$

when $f_{0}$ decreases,

$$
\Delta V_{c} \in\left(\Delta V_{b}, \Delta V_{b}+D \times \ln \left(\frac{m \times a_{c}}{a_{b}}\right)\right)
$$

Because $\Delta V_{c}=\frac{\left(a_{c}\right)^{2}}{4 b_{c}}$, where $b_{c}$ is the optimal system parameter $b$ for the current frequency. Hence,

$$
\left\{\begin{aligned}
b_{c . m i n} & =\frac{\left(a_{c}\right)^{2}}{4 \Delta V_{c . m a x}} \\
b_{c . m a x} & =\frac{\left(a_{c}\right)^{2}}{4 \Delta V_{c . m i n}}
\end{aligned}\right.
$$

where $\Delta V_{c . \text { min }}=\Delta V_{b}+D \times \ln \left(\frac{a_{c}}{m \times a_{b}}\right), \Delta V_{c . \max }=$ 
$\Delta V_{b}+D \times \ln \left(\frac{m \times a_{c}}{a_{b}}\right)$. Therefore, $b_{c} \in\left(b_{c . \min }, b_{c . \max }\right)$.

\section{Simulations}

For the input signal, we set the amplitude $A=0.1$ and frequency $f_{0}=0.01 \mathrm{~Hz}$. The signal sampling frequency $f_{s}$ $=10 \mathrm{~Hz}$, and the number of signal sample is $l=10000$. The population size of genetic algorithm $M=100$, crossover probability $p_{s}=0.6$, and mutation probability $p_{m}=0.001$. The searching ranges of system parameters $a$ and $b$ are $[0.0001 \sim 20]$ and $[0.0001 \sim 1000]$, respectively.

First, the background noise is assumed to follow zero mean Gaussian distribution with standard deviation $\sqrt{2 D}$, and let $D=1$ to verify that the SR system can improve the $S N R_{o}$. The time domain and frequency domain diagrams of the received signal and output signal are shown in Fig. 4. From Fig. 4. (a), when the noise intensity $D=1$, it is observed that, due to the noise, the received signal appears non-periodic although the pure original signal is periodic. The periodicity of received signal $r(t)$ from the time domain waveform is not obvious, and the original cosine signal $s(t)$ is completely submerged in the background noise. The spectrum amplitude of target frequency $f_{0}=0.01 \mathrm{~Hz}$ is not the biggest and smaller than that of some other frequencies in Fig. 4. (c). Therefore, the target frequency $f_{0}$ could not be detected from the received signal $r(t)$. However the time domain waveform of the output signal has obvious periodicity in Fig. 4. (b), and the spectrum amplitude of target frequency $f_{0}$ is significantly higher than that of other frequencies in Fig. 4. $(d)$. The $S N R_{i}=-27.6647$ $\mathrm{dB}$ and the $S N R_{O}=-8.6511 \mathrm{~dB}$. The $S N R_{O}$ increases by $19.0136 \mathrm{~dB}$. Therefore, the $S N R_{o}$ of weak signal with strong background noise can be increased and the target frequency can be detected by SR system.

\section{A. The performance of SR System with the CSNR Index}

We compare the performance of SR systems with the CSNR, PMV, and WPSK indexes for weak signal frequency detection. With 500 trials, the different frequency detection

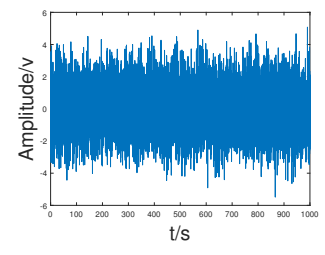

(a)

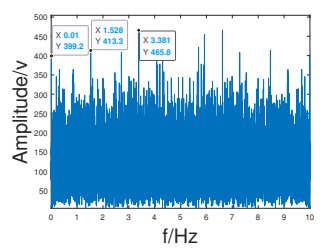

(c)

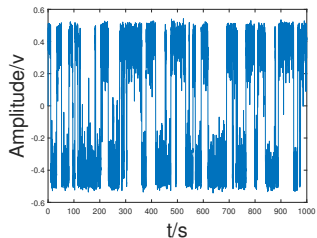

(b)

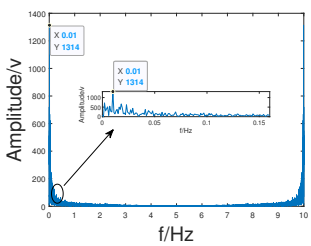

(d)
Fig. 4. (a) Received Signal in Time Domain, (b) Output Signal in Time Domain, $(c)$ Received Signal in Frequency Domain, $(d)$ Output Signal in Frequency Domain. probability $p$ of SR systems with different indexes are shown in Fig. 5, denoted by $p_{c}, p_{p}$ and $p_{w}$, respectively. The detection probability $p$ decreases with $S N R_{i}$ decreases. $p_{c}$ is higher than $p_{p}$ and $p_{w}$ when the $S N R_{i}$ ranges from -32 to $-19 \mathrm{~dB}$. This indicates that the CSNR index is more robust. $p_{c}$ and $p_{w}$ are approximate to 1 and higher than $p_{p}$ when the $S N R_{i}$ ranges from -19 to $-16 \mathrm{~dB}$. From Fig. 5, the $S N R_{O}$ of SR system with the CSNR index is improved by around $2.3 \mathrm{~dB}$ and 6.1 dB compared to the output by PMV and WPSK indexes, respectively when detection probability $p$ is around 0.9 .

We compare the sensitivity of SR systems with the CSNR, PMV, and WPSK indexes, respectively, regarding the signal frequency change. Let noise intensity $D=1$ and the input signal frequency $f_{0}$ varies from 0.01 to $0.3 \mathrm{~Hz}$ with step of 0.01 Hz. The detection results are shown in Fig. 6 and the detection probability $p$ of SR systems with the three indexes decreases with the input signal frequency increases, which verifies that the SR system is adaptive to the detection of low frequency signal. $p_{c}$ is higher than 0.8 and $p_{p}$ is between 0.5 and 0.8 . In addition, $p_{w}$ is lower than 0.5 and decreases rapidly. Therefore, the SR system with CSNR index is less sensitive to the change of signal frequency and more robust than the SR systems with PMV and WPSK indexes.

\section{B. Time Efficiency of Single Frequency Signal Detection}

Let $\tan \theta=\Delta V / x_{m}=1 / 30$ in Fig. 1. We compare the signal detection probability $p$ and time $T$ by SR systems with the different searching ranges of parameter $b$ and different input signal-to-noise ratios. The detection results are shown in Fig. 7. It indicates that the detection probability $p$ of weak signal frequency with different searching ranges of system parameter $b$ decreases with the $S N R_{i}$. The detection probability $p$ with different searching ranges of system parameter $b$ are no obvious difference basically when $S N R_{i}$ rangs from -32 to $16 \mathrm{~dB}$. From Fig. 7. (b), it is clear that the detection time $T$ with different searching ranges of system parameter $b$ increases when $S N R_{i}$ varies from -32 to $-21 \mathrm{~dB}$ and then decreases when $S N R_{i}$ varies from -21 to $-16 \mathrm{~dB}$. It indicates that when the signal amplitude $A=0.1$, frequency $f_{0}=0.01 \mathrm{~Hz}$, and the

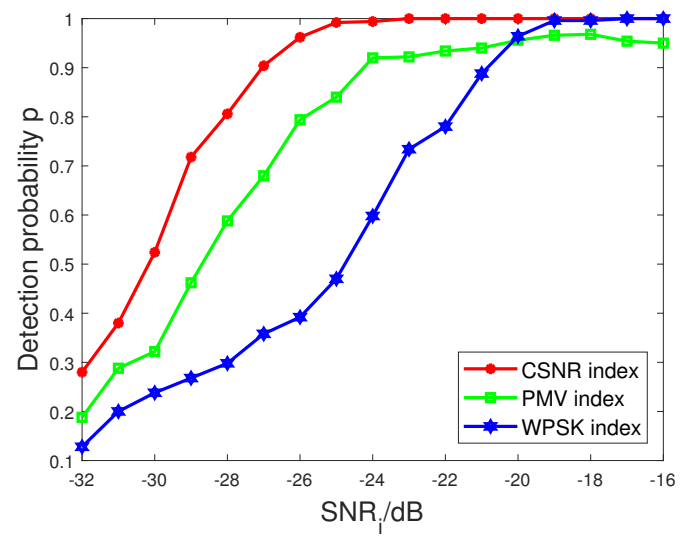

Fig. 5. The Single Frequency Signal Detection Probability by SR Systems with Different Indexes and Different Input Signal-to-Noise Ratios, where the Red, Green and Blue Curves are the Detection Probability with the CSNR, PMV and WPSK Indexes, respectively. 


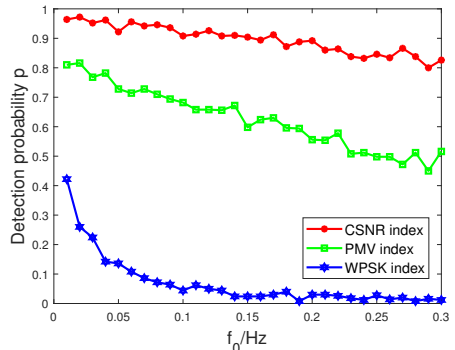

Fig. 6. The Single Frequency Signal Detection Probability by SR Systems with Different Indexes and Different Signal Frequencies, where the Red, Green and Blue Curves are Detection Probability with the CSNR, PMV, and WPSK Indexes, respectively.

$S N R_{i}=-21 \mathrm{~dB}$, the $S N R_{o}$ of SR system will be maximized. The trends of three detection time curves are similar to the $S N R_{O}$ with respect to the noise intensity $D$ (see Fig. 2).

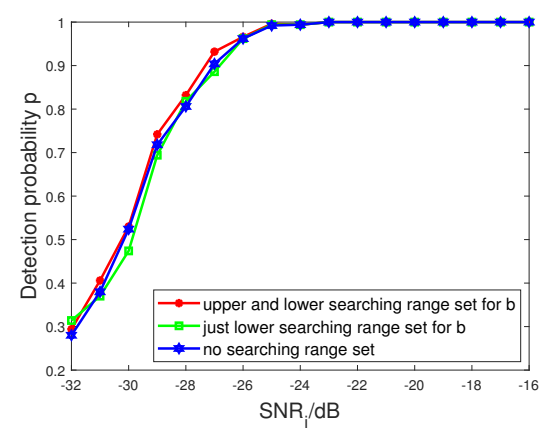

(a)

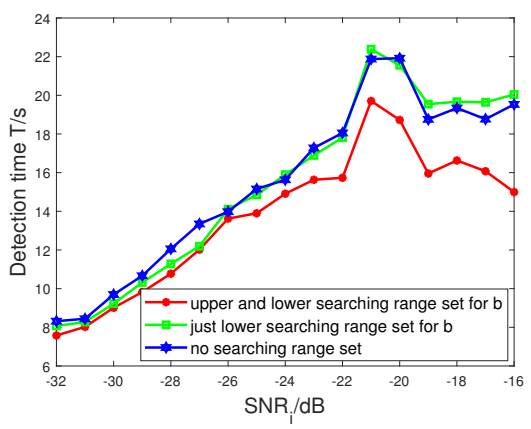

(b)

Fig. 7. With the Different Searching Ranges of Parameter $b$, the Single Frequency Signal Detection Probability and Detection Time with CSNR Index and Different Input Signal-to-Noise Ratios.

Denote the optimal noise intensity by $D_{o}$. When $D<D_{o}$, the $S N R_{O}$ of SR system increases with noise intensity $D$. In this situation, when noise intensity $D$ increases, the SR system needs more time to detect the weak signal. When $D=D_{o}$, the SR system has the maximal $S N R_{O}$ and the detection time of signal frequency should be maximized. When $D>D_{o}$, the $S N R_{O}$ of SR system decreases with the noise intensity $D$. The output signal of SR system is suboptimal and the detection time will decrease when noise intensity $D$ increases. From Fig.
7. (b), the detection time with the upper and lower searching ranges of parameter $b$ is less than that of the other searching ranges of parameter $b$ obviously. Therefore, by bounding the searching range of parameter $b$, the detection time $T$ can decrease. When the signal frequency changes with $D=1$ and $f_{0}$ varies from 0.01 to $0.3 \mathrm{~Hz}$ with step of $0.01 \mathrm{~Hz}$, the signal detection probability $p$ and time $T$ of SR systems with different searching ranges of parameter $b$ are shown in Fig. 8. It is observed that the signal frequency detection time is decreased when the method of bounding the parameter $b$ searching range is applied.

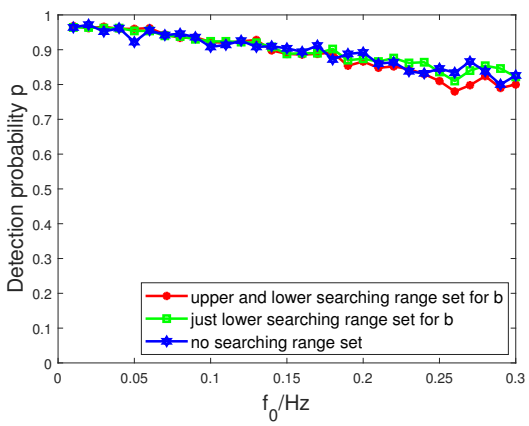

(a)

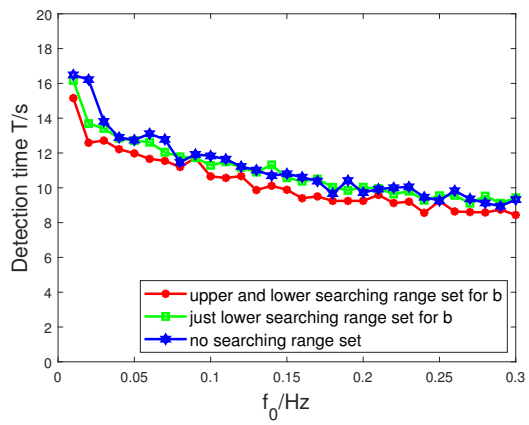

(b)

Fig. 8. With the Different Searching Ranges of Parameter $b$, the Single Frequency Signal Detection Probability and Detection Time with CSNR Index and Different Signal Frequencies.

\section{Time Efficiency of Frequency-Hopping Signal Detection}

For the input frequency-hopping signal, set amplitude $A$ $=0.1$, and the signal hopping frequency is $[0.01,0.06,0.01$, $0.02,0.04,0.08,0.05,0.10,0.15,0.13] \mathrm{Hz}$, respectively. The signal sampling frequency $f_{s}=10 \mathrm{~Hz}$, and the number of sampling points of signal for each frequency is $l=10000$. The change of signal frequency is shown in Fig. 9. (a). SNR ranges from -24 to $-16 \mathrm{~dB}$. With 100 trials, the signal detection time $T$ with different searching ranges of parameters $a$ and $b$ is shown in Fig. 9. (b), and the detection probability $p$ for each frequency is 1 . It can be concluded that the detection time for frequency-hopping signal can decrease by SR system when the method of bounding the parameters $a$ and $b$ searching ranges is applied. 


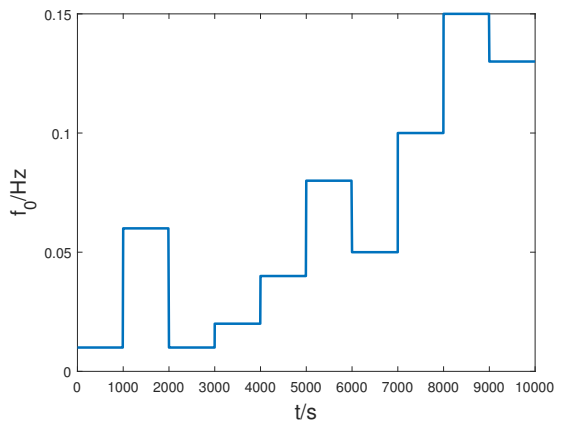

(a)

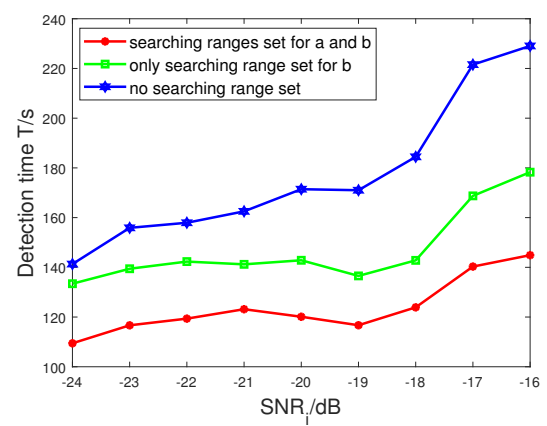

(b)

Fig. 9. (a) The Frequency of Original Frequency-Hopping Signal. (b) With the different Searching Ranges of Parameters $a$ and $b$, the

Frequency-Hopping Signal Detection Time by SR System with the CSNR Index.

\section{EXPERIMENTS}

\section{A. Single Frequency Signal Detection}

The experimental site is located at the seaside of Xinghai Park in Dalian City. The signal of a motor with a fixed frequency is used as the weak signal to be detected. A mobile phone is used as the receiving device. The signal sampling frequency $f_{s}=8000 \mathrm{~Hz}$. The motor and phone are placed below the sea surface around $0.5 \mathrm{~m}$ and the distance between them is around $2 \mathrm{~m}$. The received signal $r_{s}$ is shown in Fig. 10. $r_{s}$ is amplified by a factor $g, g=3$. We select three periods of signals $r_{i}(i=1,2,3)$ from $r_{s}$. The number of signal samples for each period is $l=10000$. The sample points are [325000 335000], [356000 366000], $\quad[450000 \sim 460000]$ for $r_{1}, r_{2}$ and $r_{3}$, respectively. The detection results are shown in Tab. I and Fig. 11. The detected frequency $f_{0}^{\prime}=0.028$ $\mathrm{Hz}$ by SR system and the actual signal frequency can be obtained by $f_{0}=f_{0}^{\prime} \times R=56 \mathrm{~Hz}$, where $R$ is the ratio of down-converting the high frequency of actual signal into a low frequency, $R=2000$. From Fig. 11, the frequency of weak signal $r_{1}$ can be detected by SR systems with CSNR, PMV, and WPSK indexes, respectively. For the output signal, the spectrum amplitude of frequency $f_{0}^{\prime}$ with the CSNR index is higher than that by each of the other two indexes. From Tab. I, the $S N R_{O}$ by SR system with the CSNR index is higher than the output by the other two indexes, and the detection time of single frequency by SR system with the upper and lower searching ranges of parameter $b$ is less than that when no searching range is set for parameter $b$ or only lower searching range is set.

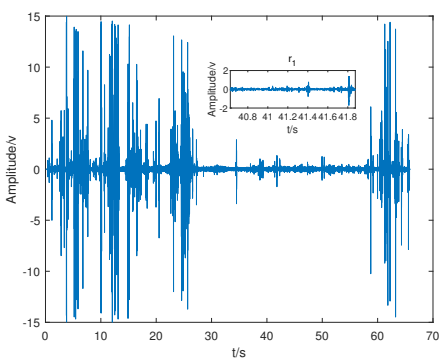

Fig. 10. Original Received Signal $r_{s}$

\section{B. Frequency-Hopping Signal Detection}

The signal of a motor with dynamic frequency change is used as the weak signal to be detected. The signal sampling frequency $f_{s}=44100 \mathrm{~Hz}$. The frequency of the motor is adjusted dynamically by a knob. The motor and phone are place below the sea surface around $0.5 \mathrm{~m}$ and the distance between them is around $4 \mathrm{~m}$. The received signal $r_{s}$ is shown in Fig. 12. $r_{s}$ is amplified by a factor $g, g=200$. We select ten periods of signals $r_{i}(i=1 \ldots 10)$ from signal $r_{s}$. The number of signal samples for each period is $l=10000$. Then the ten periods of signals are combined into a new frequency-hopping signal $s$. From Fig. 13. (a) and Fig. 14. (a), the periodicity of signal $s$ is not obvious. However, the output signals by SR systems with different parameters searching ranges show a strong periodicity (see Fig. 13. $(b),(c),(d)$ ). The time-frequency spectrum of the output signals by the SR systems with different searching ranges of system parameters $a$ and $b$ are shown in Fig. 14 . $(b),(c),(d)$, where the color brightness for each frequency in the signal spectrum indicates the magnitude of the normalized spectrum amplitude. In each of four cases, the target frequency is detected, i.e., the spectrum amplitude of the target frequency is the maximum and it is indicted by the brightest color. The frequency-hopping signal $s$ is detected in four cases: 1) frequency-hopping signal $s ; 2$ ) no searching ranges set for $a$ and $b ; 3$ ) set searching range of parameter $b$ for single frequency signal; and, 4) set searching ranges of $a$ and $b$ for frequency-hopping signal. The detection results are the same for the four cases, however the periodicity of system output signal for the case 2), 3) and 4) are more strong than case 1). The detection frequencies are $f^{\prime}=[0.063945,0.134505$, $0.055125,0.063945,0.072765,0.059535,0.14112,0.06615$, $0.090405,0.04851] \mathrm{Hz}$ by SR systems with different searching ranges of parameters $a$ and $b$, respectively. The actual signal frequencies can be obtained by $f=f^{\prime} \times R=[127.89,269.01$, $110.25,127.89,145.53,119.07,282.24,132.30,180.81,97.02]$ $\mathrm{Hz}$, where $R=2000$. The detection time of the frequencyhopping signal $s$ with different searching ranges of parameters are $307.374 \mathrm{~s}, 282.517 \mathrm{~s}$, and $226.736 \mathrm{~s}$, respectively. It indicates for frequency-hopping signal that the detection time by SR system by setting the searching ranges for $a$ and $b$ decreases by $80.638 \mathrm{~s}$ and $55.781 \mathrm{~s}$, respectively, than that produced when no searching range is set or only the searching range for parameter $b$ is set. 
(IJACSA) International Journal of Advanced Computer Science and Applications,

TABLE I. The Detection Results of Single Frequency Signal

\begin{tabular}{|c|c|c|c|c|c|c|}
\hline \multirow{2}{*}{$\begin{array}{l}\text { Different } \\
\text { indexes }\end{array}$} & \multicolumn{2}{|c|}{$r_{1}$} & \multicolumn{2}{|c|}{$r_{2}$} & \multicolumn{2}{|c|}{$r_{3}$} \\
\hline & $S N R_{o} / d B$ & $T / s$ & $S N R_{o} / d B$ & $T / s$ & $S N R_{o} / d B$ & $T / s$ \\
\hline$\overline{\operatorname{CSNR}}_{n o}$ & -9.3987 & 16.7440 & -8.2307 & 9.1730 & -9.6321 & 9.5680 \\
\hline $\mathrm{CSNR}_{l}$ & -9.7140 & 10.6470 & -8.5466 & 13.6340 & -9.8430 & 18.5040 \\
\hline $\mathrm{CSNR}_{u l}$ & -9.3512 & 8.2720 & -8.1565 & 8.5050 & -9.6743 & 8.4370 \\
\hline PMV & -9.7326 & 15.1588 & -8.6778 & 10.3360 & -9.7843 & 8.7956 \\
\hline WPSK & -9.8321 & 13.7259 & -8.8342 & 9.0453 & 10.3420 & 9.5723 \\
\hline
\end{tabular}

${ }^{a} \mathrm{CSNR}_{n o}$ is the condition with the CSNR index and the no searching range set.

${ }^{b} \mathrm{CSNR}_{l}$ is the condition with the CSNR index and the only lower searching range set for $b$.

${ }^{c} \mathrm{CSNR}_{u l}$ is condition with the CSNR index and the upper and lower searching ranges set for $b$.
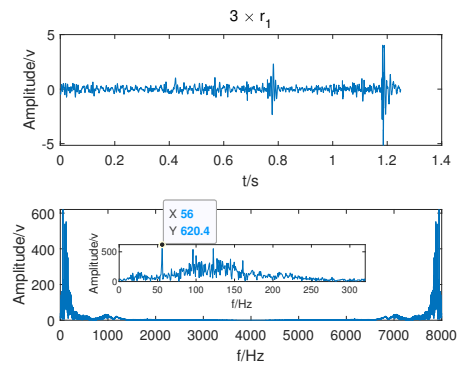

(a)
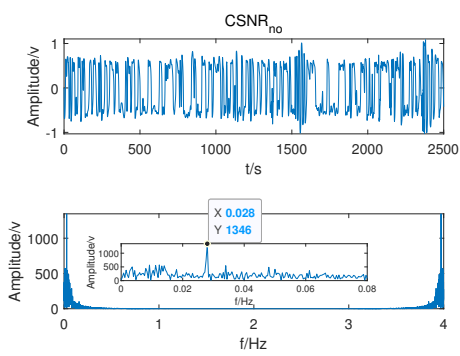

(d)
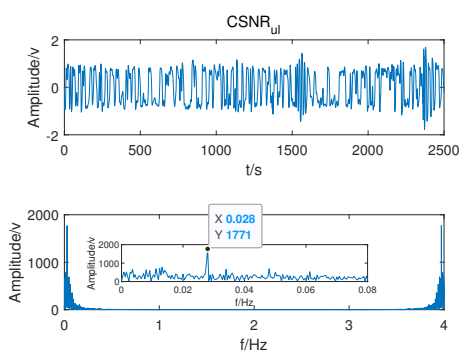

(b)
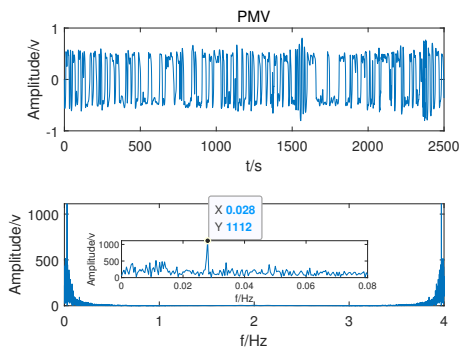

(e)
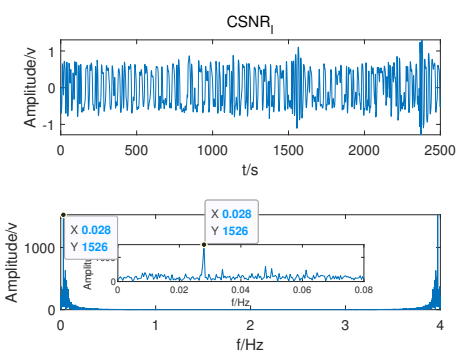

(c)
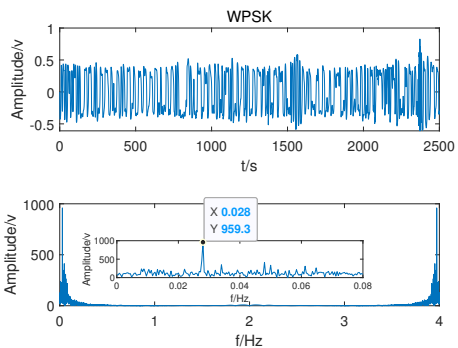

(f)

Fig. 11. The Detection Results of Single Frequency Signal $r_{1}$ by SR Systems with Different Indexes and Searching Ranges of Parameter $b$.

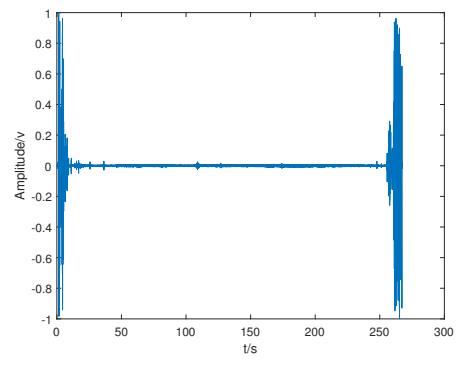

Fig. 12. Original Received Signal $r_{s}$

\section{RESUlTS AND Discussion}

The performance of weak signal detection by SR system with the CSNR index is verified by simulations and experiments. The frequency detection probability of SR systems with CSNR, PMV, and WPSK indexes are denoted by $p_{c}, p_{p}$ and $p_{w}$, respectively. In simulations, $p_{c}$ of weak signal with the CSNR index is 1 when the $S N R_{i}$ is higher than $-26 \mathrm{~dB}$, and $p_{c}$ is as high as 0.9 when $S N R_{i}=-27.5 \mathrm{~dB} . p_{c}$ is higher than $p_{p}$ and $p_{w}$ when $S N R_{i}$ varies from -32 to $-19 \mathrm{~dB} . p_{c}$ and $p_{p}$ are approximate to 1 and higher than $p_{w}$ when $S N R_{i}$ is $[-19 \sim-16] \mathrm{dB}$. When $S N R_{i}=-26 \mathrm{~dB}$, the performance of SR system with the CSNR index is insensitive to the change of input signal frequency. $p_{c}$ is higher than 0.8 and $p_{p}$ is between 0.5 and 0.8 when the input signal frequency $f_{0}$ varies from 0.01 to $0.3 \mathrm{~Hz}$ with step of $0.01 \mathrm{~Hz}$. However, $p_{w}$ is lower than 0.5 and $p_{w}$ decreases rapidly. With the searching range set for system parameter $b$, the detection time of single frequency signal with the CSNR index decreases. The $S N R_{o}$ of SR system with CSNR index is higher than the output by PMV and WPSK indexes in the same condition. For frequency-hopping signal, with the searching ranges set for system parameters $a$ and $b$, when $S N R_{i}$ varies from -24 to $-16 \mathrm{~dB}$, the signal detection time will decrease significantly. The experimental results are consistent with the simulation results. 


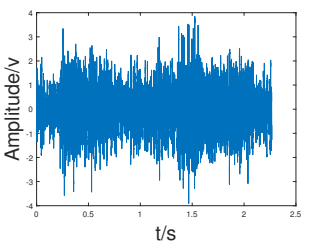

(a)

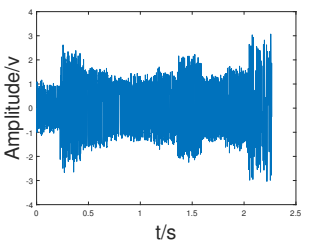

(c)

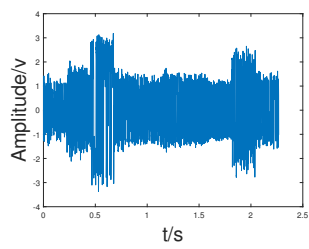

(b)

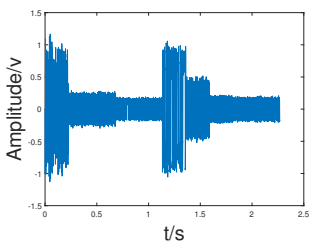

(d)
Fig. 13. The Signal in Time Domain. (a) Frequency-Hopping Signal $s,(b)$ the Output Signal with no Searching Range Set, $(c)$ the Output Signal with

Searching Range Set for Parameter $b$ only, $(d)$ the Output Signal with Searching Ranges Set for Parameters $a$ and $b$.

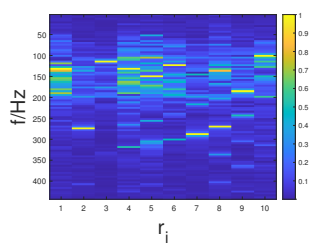

(a)

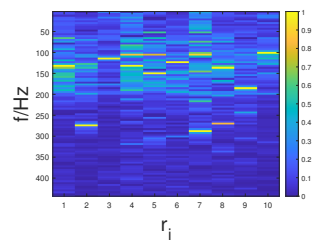

(c)

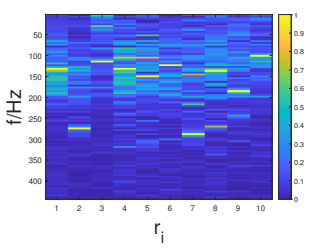

(b)

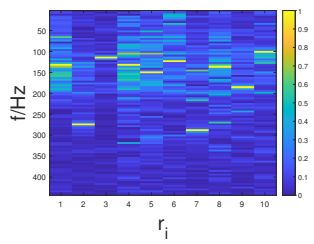

(d)
Fig. 14. The Signal in Time-Frequency Spectrum. The X-Label Represents Signal $r_{i}(i=1 \ldots 10)$ and Y-Label Represents the Output Signal Frequency (from 0 to $500 \mathrm{~Hz}$ ). (a) Frequency-Hopping Signal $s,(b)$ the Output Signal with no Searching Range Set, $(c)$ the Output Signal with Searching Range Set for Parameter $b$ only, $(d)$ the Output Signal with Searching Ranges set for Parameters $a$ and $b$.

\section{FUtURE PLANS AND IMPROVEMENTS}

The future plans are summarized as follows. First, the machine learning method can be combined with the genetic algorithm in this paper to further improve the detection time efficiency of unknown signals. Second, we can use underwater sonar array to improve the signal detection probability. The experimental verification part needs to be improved. I hope to conduct experiments in deep sea in the future to obtain more diverse and accurate underwater signals.

\section{CONCLUSION}

In this paper, a new index, called CSNR, is proposed to detect the frequency of unknown underwater signals based on SR theory with genetic algorithm, and the method of bounding searching ranges of system parameters $a$ and $b$ is presented to reduce the detection time. The performance of weak signal detection by SR system with the CSNR index is verified by simulations and experiments. The frequency detection probability of SR systems with CSNR, PMV, and WPSK indexes are denoted by $p_{c}, p_{p}$ and $p_{w}$, respectively. The results show that $p_{c}$ is higher than $p_{p}$ and $p_{w}$. In addition, the performance of SR system with the CSNR index is insensitive to the change of input signal frequency. With the searching ranges set for system parameters $a$ and $b$, the signal detection time will decrease significantly in simulations and experiments. In conclusion, the SR system with the proposed CSNR index and parameter searching ranges outperforms SR systems with PMV and WPSK indexes in terms of detection probability and detection time. Therefore, the contributions proposed in this paper are of positive significance to the detection of underwater weak signals in practical applications.

\section{ACKNOWLEDGMENT}

This research did not receive any specific grant from funding agencies in the public, commercial or nonprofit sectors. My sincere acknowledgment goes to Dr. Li Xiukui.

\section{REFERENCES}

[1] Li, X., Tan., W. Design and Implementation of IIR Multi-path Filter for SSVEP Based on MATLAB. 2019 IEEE 4th International Conference on Image, Vision and Computing (ICIVC), pp. 83-87, 2019.

[2] M, L., A., K., High speed FIR adaptive filter for RADAR applications. 2016 International Conference on Wireless Communications, Signal Processing and Networking (WiSPNET), Chennai, India, pp. 2118-2122, 2016

[3] Dong, H.,Wang, H., Shen, X., Huang, Z., Ma., S. Detection of underwater weak signal via matched stochastic resonance. OCEANS 2017 Aberdeen, pp. 1-7, 2017.

[4] R. Lang, F.G. X. Li, Yang., L. Re-scaling and adaptive stochastic resonance as a tool for weak gnss signal acquisition. Journal of Systems Engineering and Electronics. 27(2), 290-296, 2016.

[5] Lucarini, V. Stochastic resonance for nonequilibrium systems. Phys. Rev.E. 100, 062124, 2019. https://doi.org/10.1103/PhysRevE.100.062124

[6] Bordi, I., Sutera., A. Stochastic perturbation in meteorology. Waves in Random Media. 10(3), 1-30, 2000. https://doi.org/10.1088/0959$7174 / 10 / 3 / 201$

[7] Liu, W., Wang, Y., Liu, X. Weak thruster fault detection for auv based on stochastic resonance and wavelet reconstruction. Journal of Central South University. 23(11), 2883-2895, 2016.

[8] Han, D., li, P., An, S., Shi., P. Multi-frequency weak signal detection based on wavelet transform and parameter compensation band-pass multi-stable stochastic resonance. Mechanical Systems and Signal Processing. 70-71, 995-1010, 2015. https://doi.org/10.1016/j.ymssp.2015.09.003

[9] Xuefang Xu, Y.L. An incorrect data detection method for big data cleaning of machinery condition monitoring. Industrial Electronics. 67(3), 2326-2336, 2020.

[10] Shi, P., Yuan, D., Han, D., Zhang, Y., Fu., R. Stochastic resonance in a time-delayed feedback tristable system and its application in fault diagnosis. Journal of Sound and Vibration. 424, 1-14, 2018. https://doi.org/10.1016/j.jsv.2018.03.007

[11] Wang, Y., Jiao, S., Zhang, Q., Lei, S., Qiao., X. A weak signal detection method based on adaptive parameter-induced tri-stable stochastic resonance. CHINESE JOURNAL OF PHYSICS. 56, 1187-1198, 2018. https://doi.org/10.1016/j.cjph.2018.04.002 
[12] Qi, W., Liu, Y., Guo, S., Wang, X., Guo., Z. An adaptive data detection algorithm based on intermittent chaos with strong noise background. Neural Comput and Applic. 32, 16755-16762, 2018. https://doi.org/10. 1007/s00521-018-3839-9

[13] Reda, H.T., Mahmood, A., Diro, A. Firefly-inspired stochastic resonance for spectrum sensing in cr-based iot communications. Neural Comput and Applic. 32, 16011-16023, 2019. https://doi.org/10.1007/s00521019-04584-0

[14] Liu, W., Liu, Z. Magnetic anomaly signal detection using parallel monostable stochastic resonance system. IEEE Access. 8, 162230-162237, 2020. https://doi.org/10.1109/ACCESS.2020.3020881

[15] Guo, W., Zhou, Z., Chen, C. Cascaded and parallel stochastic resonance for weak signal detection and its simulation study. 2016 Prognostics and System Health Management Conference (PHM-Chengdu), 2016, pp. 16, doi: 10.1109/PHM.2016.7819839, 2016.

[16] Liu, H.G., Liu, X.L., Yang, J.H. Detecting the weak high-frequency character signal by vibrational resonance in the duffing oscillator. Nonlinear Dyn. 89(4), 2621-2628, 2017. https://doi.org/10.1007/s11071017-3610-2

[17] Chen, L., Zhang, Y., Feng, A., Xu, Z., Li, B., Shen., H. A new model of stochastic resonance used in weak signal detection. AppliedMechanics and Materials. 43, 229-232, 2010. https://doi.org/10.4028/www.scientific.net/AMM.43.229

[18] Liu, J., Leng, Y., Lai, Z., Fan., S. Multi-frequency signal detection based on frequency exchange and re-scaling stochastic resonance and its application to weak fault diagnosis. Sensors. 18(5), 1325-1325, 2018. https://doi.org/10.3390/s18051325

[19] Shu-Yao, J., Fei, Y., Ke-Yu, C., En, C. Application of stochastic resonance technology in underwater acoustic weak signal detection. OCEANS 2016 - Shanghai, 2016, pp. 1-5, doi: 10.1109/OCEANSAP.2016.7485567 2016.

[20] Gauthier, P.-A. et al., A. Acoustical inverse problems regularization: Direct definition of filter factors using signal-to-noise ratio. Journal of Sound and Vibration. 333(3), 761-773, 2014.

[21] Cheng, W., Xua, X., Ding, Y., Sun., K. Stochastic resonance in a singlewell potential and its application in rolling bearing fault diagnosis. Review of Scientific Instruments. 91(6), 761-773, 2020.

[22] Li, J., Chen, X., He., Z. Adaptive stochastic resonance method for impact signal detection based on sliding window. Me- chanical Systems and Signal Processing. 36(2), 240-255, 2013. https://doi.org/10.1016/j.ymssp.2012.12.004

[23] Xiang, J., Zhong, Y., Gao., H. Rolling element bearing fault detection using ppca and spectral kurtosis. Measurement. 75, 180-191, 2015. https://doi.org/10.1016/j.measurement.2015.07.045

[24] Morales., C.A. Comments on the mac and the nco, and a linear modal correlation coefficient. Journal of Sound and Vibration. 282(1), 529-537, 2004. https://doi.org/10.1016/j.jsv.2004.04.011

[25] Wang, J., He, Q., Kong, F. Adaptive multiscale noise tuning stochastic resonance for health diagnosis of rolling element bearings. Instrumentation and Measurement. 64(2), 564-577, 2015.

[26] Huang, Z., Wang, H., Dong, H., Jian, S. Detection of Underwater Weak Signals Based on Stochastic Resonance Multi-Measures Fusion.2018 OCEANS - MTS/IEEE Kobe Techno-Oceans (OTO), pp. 1-6, doi:10.1109/OCEANSKOBE.2018.8559075, 2018.

[27] Huang, D., Yang., J. Recovering an unknown signal completely submerged in strong noise by a new stochastic resonance method. Communications in Nonlinear Science and Numerical Simulation. 66, 156-166, 2018. https://doi.org/10.1016/j.cnsns.2018.06.011

[28] Wang, X., Huang. Adaptive stochastic resonance method based on quantum genetic algorithm and its application in dynamic characteristic identification of bridge gnss monitoring data. IEEE Access. 8, 113994-114009, 2020. https://doi.org/10.1109/ACCESS.2020.3002889

[29] Li, J., Li, M., Zhang., J. Rolling bearing fault diagnosis based on time-delayed feedback monostable stochastic resonance and adaptive minimumentropy deconvolution. Journal of Sound and Vibration. 401, 139-151, 2017. https://doi.org/10.1016/j.jsv.2017.04.036

[30] Yan Ren, J.H. Research on fault feature extraction of hydropower units based on adaptive stochastic resonance and fourier decomposition method. Shock and Vibration. 2021, 2021. https://doi.org/10.1155/ 2021/6640040

[31] Qiu, Y., Yuan, F., Ji, S., Cheng., E. Stochastic resonance with reinforcement learning for underwater acoustic communication signal. Applied Acoustics. 173, 2021. https://doi.org/10.1016/j.apacoust.2020.107688

[32] Li, J., Li, M., Zhang., J. Research on detection method of multifrequency weak signal based on stochastic resonance and chaos characteristics of duffing system. CHINESE JOURNAL OF PHYSICS. 64, 333-347, 2020. https://doi.org/10.1016/j.cjph.2019.12.001 